\title{
MTHFD2 facilitates breast cancer cell proliferation via the AKT signaling pathway
}

\author{
JUN HUANG $^{1 *}$, YINYIN QIN ${ }^{2,3^{*}}$, CANFENG LIN ${ }^{4 *}$, XIAOGUANG HUANG ${ }^{4}$ and FEIRAN ZHANG ${ }^{5}$ \\ ${ }^{1}$ Department of General Thoracic Surgery; ${ }^{2}$ State Key Laboratory of Respiratory Disease, \\ National Clinical Research Center for Respiratory Disease, Guangzhou Institute of Respiratory Health, The First Affiliated \\ Hospital of Guangzhou Medical University; ${ }^{3}$ Pulmonary and Critical Care Medicine (PCCM), \\ Shunde Affiliated Hospital of Guangzhou Medical University; ${ }^{4}$ Department of Oncology, Shantou Central Hospital, \\ Jinping, Shantou, Guangzhou 515000; ${ }^{5}$ Department of General Surgery, The First Affiliated Hospital \\ of Shantou University Medical College, Jinping, Shantou, Guangdong 515041, P.R. China
}

Received January 19, 2020; Accepted October 20, 2020

DOI: $10.3892 /$ etm.2021.10135

\begin{abstract}
MTHFD2 is a folate-coupled mitochondrial metabolic enzyme which has been extensively studied in breast cancer; however, its molecular functions in this cancer remain unclear. The current study aimed to reveal the underlying mechanism of breast cancer. MTHFD2 expression status and prognostic value were determined using the Gene Expression Profiling Interactive Analysis database. To determine the function of MTHFD2 in breast cancer, MCF-7 cells with stable overexpression of Flag-MTHFD2 or depletion of MTHFD2 were generated. Cell Counting Kit-8 and colony formation assays were used to examine the effect of MTHFD2 overexpression or knockout on MCF-7 cell proliferation and clonogenicity, respectively. Luciferase reporter and an AKT inhibitor (GSK6906) analysis were carried out to investigate the effect of MTHFD2 on the AKT signaling pathway. The results demonstrated that MTHFD2 expression level was higher in breast cancer tissues compared with adjacent normal tissues. Furthermore, patients with high MTHFD2 expression had significantly poorer overall survival compared with patients with low MTHFD2 expression. In addition, ectopic expression of MTHFD2 promoted the tumorigenic properties of MCF-7 cells, including proliferation and clonogenicity. Conversely, depletion of MTHFD2 had the opposite effect on the malignant properties of MCF-7 cells. Luciferase reporter demonstrated that MTHFD2 can significantly increase the ATK
\end{abstract}

Correspondence to: Professor Feiran Zhang, Department of General Surgery, The First Affiliated Hospital of Shantou University Medical College, 57 Changping Road, Jinping, Shantou, Guangdong 515041, P.R. China

E-mail: feiran.z@126.com

*Contributed equally

Key words: MTHFD2, breast cancer, proliferation, AKT luciferase density. Furthermore, the Akt inhibitor GSK690693 significantly decreased the increased clonogenicity caused by MTHFD2 overexpression in MCF-7 cells. Taken together, the findings from the present study suggested that MTHFD2 may serve a protumor role in the malignancy of breast cancer by activating the AKT signaling pathway. These results provide an alternative theoretical foundation that could help the development of MTHFD2-targeted breast cancer treatment.

\section{Introduction}

Breast cancer is the most common cause of cancer-associated mortality and the second most frequently diagnosed malignancy in women, accounting for 0.627 million deaths and 2.088 million new cases worldwide in $2018(1,2)$. Prevention and early detection interventions have been used and have significantly contributed to outcome improvements and increased survival in patients with breast cancer (3). However, extensive evidence has demonstrated that gene-related risk factors and genetic alternations serve critical roles in the occurrence and progression of breast cancer (4), and have partially contributed to a sharp increase in the mortality rate of breast cancer in the last 40 years (5). A comprehensive understanding of the genetics and molecular pathogenesis of breast cancer is therefore crucial for clinical diagnosis and treatment.

MTHFD2 is a folate-coupled mitochondrial metabolic enzyme characterized by methylenetetrahydrofolate dehydrogenase and cyclohydrolase activity (6). This mitochondrial enzyme has been reported to actively participate in folate one-carbon metabolism and tetrahydrofolate (THF) cofactor cycling, which supply important precursors to maintain cell viability and proliferation $(7,8)$. It was demonstrated that suppression of MTHFD2 inhibits methylation reactions (7), decreases protein synthesis (9) and disrupts redox homeostasis $(6,10)$, which may ultimately result in significant changes in cellular metabolic phenotype. Because MTHFD2 expression was reported as a key participant in the metabolic reprogramming in tumors (7) and a predictive factor of poor prognosis in patients with various types of cancer $(8,11-13)$, including 
breast cancer (14), MTHFD2 has received increased attention recently and might be considered as a potential anticancer target. Numerous studies have also demonstrated a strong association between tumor cell phenotype and MTHFD2 expression status $(13,15,16)$.

In addition to the enzymatic activity of MTHFD2 in metabolic remodeling, certain non-enzymatic activities have been reported. Sheppard et al (17) demonstrated that MTHFD2 is not only a mitochondrial enzyme but also a nuclear protein implicated in DNA synthesis, and that MTHFD2 overexpression confers tumor cell-sustaining proliferative capacity independently of dehydrogenase activity, suggesting that MTHFD2 might likely regulate cell proliferation in a non-enzymatic manner. In non-small-cell lung cancer, MTHFD2 was also reported to display non-enzymatic function, where MTHFD2 silencing impaired tumor proliferation by modulating cell cycle-associated genes (11). Furthermore, it has been demonstrated that cellular mediators of signal transduction are integrated in tumor cell metabolic reprogramming to support metabolic autonomy (18). Subsequently, it could be hypothesized that MTHFD2 may serve different metabolic regulatory roles in breast cancer malignancy in a non-enzymatic manner.

AKT activation is known to be involved in the regulation of metabolic fluxes different from canonical allosteric mechanisms of pathway regulation, and to modulate complementary aspects of cellular metabolism (18). The present study examined the impact of MTHFD2 on the proliferation and colony formation ability of MCF-7 cells. In addition, the underlying molecular mechanisms of MTHFD2 in breast cancer carcinogenesis and progression were investigated.

\section{Materials and methods}

Cell culture. MCF-7 cell line and 293T cells were purchased from the China Center for Type Culture Collection. All cells were cultured in DMEM medium (Invitrogen; Thermo Fisher Scientific, Inc.) supplemented with $10 \%$ FBS and $100 \mathrm{U} / \mathrm{ml}$ penicillin-streptomycin and placed at $37^{\circ} \mathrm{C}$ in a humidified incubator containing 5\% $\mathrm{CO}_{2}$. The MCF-7 cell line was authenticated by STR profiling.

Plasmid construction and transfection. For overexpressing the candidate gene, PCR-amplified MTHFD2 cDNA was amplified from MCF-7 cells by Tianyihuiyuan and inserted into a PHAGE puro retrovirus vector (Addgene, Inc.) with a Flag tag by BamHI and NotI (Thermo Fisher Scientific, Inc.) digestion, resulting in MTHFD2-Flag PHAGE puro. 293T cells $\left(2 \times 10^{5}\right)$ were transduced with $1.3 \mu \mathrm{g}$ MTHFD2-Flag PHAGE puro or $1.3 \mu \mathrm{g}$ the empty control vector as previously described (19) for

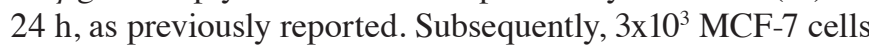
were infected with $10 \mu \mathrm{l}$ filtered virus supernatants following by a dual puromycin selection $(1 \mu \mathrm{g} / \mathrm{ml}, 7$ days). Western blotting and reverse transcription quantitative (RT-q) PCR were used to confirm MTHFD2 overexpression. MTHFD2 primer (Tianyihuiyuan) were as follows: Forward primer GGATCC AGATCAAGCAGGAAGTGCGG; reverse primer GTCTCA CTGTTGATTCCCACACCGGCG.

MTHFD2-deficient MCF-7 cells were generated by CRISPR/Cas9-mediated genome editing technology. The single guide RNA (sgRNA) was designed via (http://sam.genome-engineering.org/), and the sequence of sgRNA targeted MTHFD2 exon 1 is was 5'-GCCACACCT GAGTGTGATCC-3' as described previously (19). Briefly, 2,500 MCF-7 cells per well were seeded at 6 -well plate to $60-70 \%$ confluence and were infected with $350 \mathrm{ng}$ MTHFD2 sgRNA or wild-type Cas9 plasmids (Addgene, Inc.; cat. no. 42230) using Lipofectamine ${ }^{\circledR} 2000$ (Thermo Fisher Scientific, Inc.) for 2 days. The MTHFD2 knockout clones were screened with $1 \mu \mathrm{g} / \mathrm{ml}$ puromycin for 7 days. MTHFD2 loss was confirmed in MCF-7 cells by western blotting. Two knockout cell clones, MTHFD2 $2^{-/ 1 \#}$ and MTHFD2 $2^{-/ 2 \#}$, were chosen for subsequent experiments. The uninfected cells were used as the control.

$R T$ - $q P C R$. RNA isolation from MCF-7 cells was performed using via a High Pure RNA isolation kit [Roche Diagnostics (Shanghai) Co., Ltd.]. The RNA was reversely transcribed into cDNA using the SuperScript VILO cDNA kit (Thermo Fisher Scientific, Inc.). Thermocycling conditions of RT-PCR is $95^{\circ} \mathrm{C}$ for $5 \mathrm{~min}$, followed by 40 cycles of $95^{\circ} \mathrm{C}$ for $10 \mathrm{sec}, 60^{\circ} \mathrm{C}$ for $20 \mathrm{sec}$ and $72^{\circ} \mathrm{C}$ for $30 \mathrm{sec}$. MTHFD2 primers used were as follows: Forward, 5'-GAGCTTTGGAGAAACCAGCC-3'and reverse 5'-CAGAAGAACGAGGACGGAGG-3'. Quantitative PCR was performed using Brilliant II SYBR-Green RT-qPCR kit (Merck \& Co., Inc.). qPCR thermocycling conditions were as follows: $94^{\circ} \mathrm{C}$ for $5 \mathrm{~min}$; 40 cycles of $95^{\circ} \mathrm{C}$ for $20 \mathrm{sec}$; and $72^{\circ} \mathrm{C}$ for $30 \mathrm{sec}$. Gene expression values were analyzed with $2^{-\Delta \Delta \mathrm{Cq}}$ method (20).

Cell proliferation and colony formation assay. To determine the role of MTHFD2 in breast cancer malignancy, in vitro functional assays were performed to compare the effect of MTHFD2 overexpression and MTHFD2 deficiency in MCF-7 cells.

Cell proliferation was determined using the Cell Counting Kit-8 (CCK-8; Abmole Bioscience, Inc.). Briefly, cells were seeded into 96-well plates at the density of 1,000 cells per well for 1, 3 and 5 days. Cells were incubated with CCK-8 and incubated at $37^{\circ} \mathrm{C}$ for $2 \mathrm{~h}$. Absorbance was read at $450 \mathrm{~nm}$ using a microplate reader.

For the colony formation assay, $2 \times 10^{2}$ cells were seeded into six-well plates for 14 days at $37^{\circ} \mathrm{C}$ and $5 \% \mathrm{CO}_{2}$. Methanol and $0.1 \%$ crystal violet were used for fixation and staining of colonies at room temperature, respectively. The number of colonies was imaged using a camera [DSC-HX90; SONY (China), Co. Ltd.] and quantified.

Luciferase assay. Since AKT is an oncogenic checkpoint in cancer cell metabolism (18), a luciferase assay was performed to examine the effect of MTHFD2 expression on the transcriptional activity of AKT. An AKT luciferase reporter plasmid was purchased from Yeasen Biotech Co., Ltd. and used for dual-luciferase reporter assays. Briefly, 293T cells were seeded at the density of $2 \times 10^{5}$ cells/well in a 24 -well plate. Once the cells had reached $85-90 \%$ confluence, they were transfected using Lipofectamine ${ }^{\circledR} 2000$ with 200 ng pAKT-luc reporter plasmids or $10 \mathrm{ng}$ pRL-TK plasmids (internal control) and then co-transfected with MTHFD2-Flag plasmids (0, 100, 200 and $400 \mathrm{ng}$ ). Two days post-transfection, cells were lysed with TRIzol ${ }^{\circledR}$ (Invitrogen; Thermo Fisher Scientific, Inc.) and the luminescence activity of AKT was measured using a Dual-Luciferase 
Reporter Assay System (Promega Corporation) following the manufacturer's protocol. Subsequently, the luminescence activity of AKT in MTHFD2 ${ }^{-/}$and normal MCF-7 cells was analyzed. Firefly luciferase activity was normalized to Renilla luciferase activity.

Drug treatment. For AKT inhibition studies, MCF-7 cells at $75-85 \%$ confluence were treated with the AKT inhibitor GSK69069 (10 nM; MedChemExpress) for $24 \mathrm{~h}$ at $37^{\circ} \mathrm{C}(21)$. Subsequently, a colony formation assay was performed in MCF-7 cells (Ctrl) and MTHFD2-overexpressing cells treated or not with GSK690693 (MTHFD2 or MTHFD2 + GSK69069).

Western blotting. MCF-7 cells were ultrasonically lysed in RIPA buffer (Sigma-Aldrich; Merck KGaA) and the collected supernatant was subjected to qualification with BCA methods. Proteins $(20 \mu \mathrm{g})$ were separated by $15 \%$ SDS-PAGE and transferred onto PVDF membranes. Membranes were incubated with $5 \%$ skim milk for $1 \mathrm{~h}$ at room temperature and with primary antibodies against MTHFD2 (1:1,000; cat. no. PA5-28169; Thermo Fisher Scientific, Inc.), Flag (1:1,000; cat. no. A02010; Abbkine Scientific Co, Ltd.), and GAPDH (1:1,000; cat. no. K106390M; Beijing Solarbio Science \& Technology Co., Ltd.) at $4^{\circ} \mathrm{C}$ for $12 \mathrm{~h}$. Membranes were then incubated with secondary anti-rabbit or anti-mouse antibodies (1:1,000; cat. nos. A32723 and A32731; Thermo Fisher Scientific, Inc.) for $1 \mathrm{~h}$ at room temperature. Bands were detected using HRP Color Development Solution. (Invitrogen; Thermo Fisher Scientific, Inc.).

Bioinformatics analysis. MTHFD2 expression data of 1,085 Breast invasive carcinoma (BRCA) tissues and 291 adjacent normal tissues from the GEPIA database (22) (http://gepia. cancer-pku.cn/) were analyzed by unpaired Student's t-test with a cutoff value of ${ }^{* *} \mathrm{P}<0.01$. The overall survival (OS) data for MTHFD2 were also analyzed via GEPIA using the Kaplan Meier method (cut-off: $50 \%$ ), plotting the survival curves on the basis of the MTHFD2 median values.

Statistical analysis. GraphPad Prism version 8 (GraphPad Software, Inc.) was used for statistical analysis, and data are expressed as the means \pm standard error of the mean. Comparison between two groups was carried out using Student's t-test, and comparison among multiple groups was performed using ANOVA followed by Dunnett's post hoc test. Data were representative of three independent experiments. $\mathrm{P}<0.05$ was considered to indicate a statistically significant difference.

\section{Results}

MTHFD2 expression is increased in breast cancer tissues and associated with poor prognosis. To determine whether MTHFD2 is involved in breast cancer progression and prognosis, the GEPIA database containing 1,080 breast cancer tissues and 291 adjacent non-tumor tissues was used to analyze MTHFD2 expression levels. The results demonstrated that MTHFD2 expression level was higher in breast cancer tissues compared with adjacent tissues though the difference was not significant (Fig. 1A). Furthermore, the results from
Kaplan-Meier and Log-rank test analyses were carried out to evaluate the prognostic value of MTHFD2, and the results indicated that patients with high MTHFD2 expression had a worse prognosis compared with patients with low MTHFD2 expression (Fig. 1B). Taken together, these findings suggest that MTHFD2 may serve a notable role in breast cancer progression.

MTHFD2 overexpression promotes MCF-7 cell proliferation and clonogenicity. To evaluate the pathogenic role of MTHFD2 in breast cancer cells, MCF-7 cells stably expressing Flag-MTHFD2 were established. The results from western blotting and RT-qPCR confirmed the successful overexpression of MTHFD2 in vitro (Fig. 2A and B). Furthermore, MCF-7 cells stably expressing MTHFD2-Flag displayed a higher proliferation rate and colony formation ability compared with control cells (Fig. 2C-E). These data suggest that MTHFD2 overexpression may promote breast cancer cell proliferation and clone formation ability in breast cancer.

MTHFD2 knockout weakens the proliferation and colony formation ability of MCF-7 cells. The effect of MTHFD2 knockout on proliferation of MCF-7 cells was assessed. The efficiency of MTHFD2-knockout by CRISPR/Cas9-mediated genome was confirmed by western blotting (Fig. 3A). Furthermore, MTHFD2 deficiency markedly retarded MCF-7 cell proliferation (Fig. 3B), as well as MCF-7 cell colony formation ability (Fig. 3C and D). These data suggest that MTHFD2 knockout may inhibit proliferation and viability of breast cancer in vitro.

MTHFD2 activates the AKT signaling pathway. Since AKT is involved in oncogenic signaling and cancer metabolism (18), the relative luciferase activity of AKT modulated by MTHFD2 was quantified using dual-luciferase assays. The results demonstrated that AKT transcriptional activity was enhanced in an MTHFD2 expression-dependent manner (Fig. 4A), suggesting that AKT may be a direct regulator of MTHFD2 in breast cancer cells. The transcriptional activity of AKT was also evaluated in normal and MTHFD2-deficient MCF-7 cells. The results demonstrated that MTHFD2 knockout yielded an inhibitory effect on the AKT-mediated luciferase reporter expression. Subsequently, an AKT inhibitor, GSK69069, was used to inhibit AKT signaling in order to verify whether suppressing AKT signaling could counteract MCF-7 cell clonogenicity caused by MTHFD2 overexpression. As presented in Fig. 4C and D, the colony formation ability increase following MTHFD2-overexpression was abolished when cells were treated with GSK69069. These results indicate that MTHFD2 may stimulate MCF-7 cell proliferation and colony formation ability via the AKT signaling pathway.

\section{Discussion}

High expression of MTHFD2 has been considered as a prognostic indicator in breast cancer, and results from MTHFD2 silencing in vitro have highlighted its metabolic role in breast cancer malignancy (20). However, a previous study reported that there is no association between tumor cell proliferation and the enzymatic activity of MTHFD2 (13); however, MTHFD2 
A

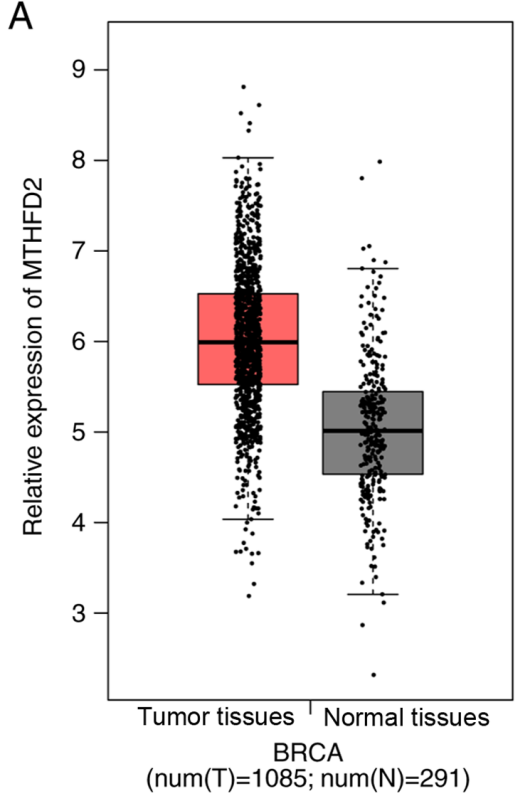

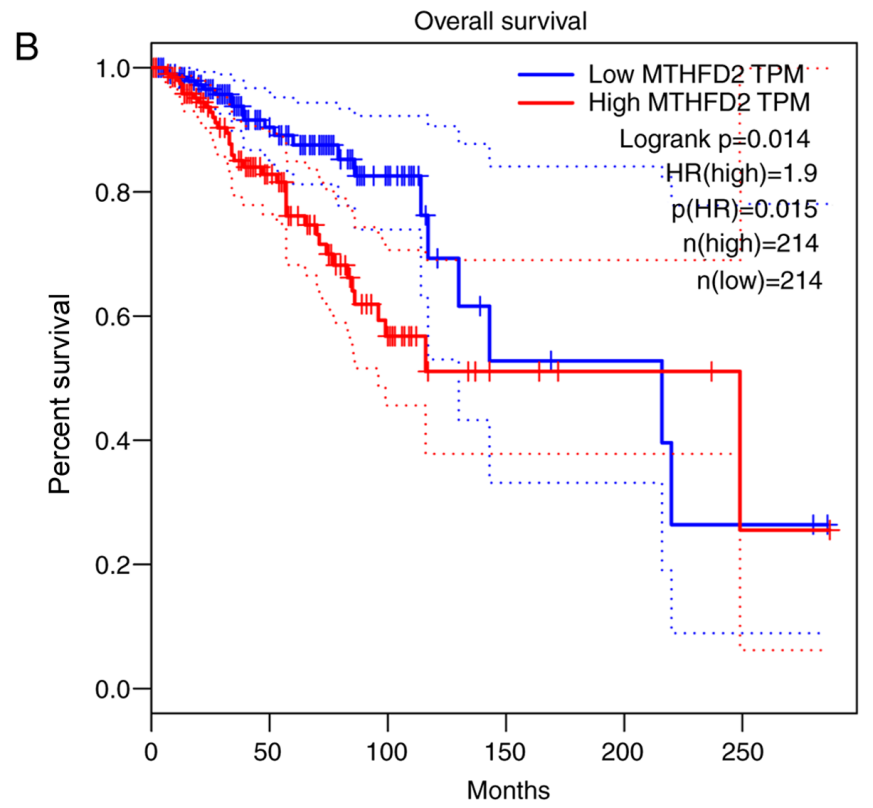

Figure 1. MTHFD2 expression in breast cancer. (A) GEPIA database analysis demonstrated that MTHFD2 was overexpressed in 1,085 Breast invasive carcinoma (BRCA) compared with 291 adjacent normal tissues. (B) Prognostic significance of MTHFD2 expression in patients with breast cancer. Kaplan-Meier analysis was performed to estimate the association between MTHFD2 expression level and overall survival time in patients included in the TCGA dataset. BRCA, breast cancer; TCGA, The Cancer Genome Atlas.

may still be involved in signal transduction related to cell proliferation (17). In the present study, the high expression of MTHFD2 in breast cancer tissues, and its prognostic role in patients with breast cancer, was verified using the GEPIA database. Furthermore, this study demonstrated that MTHFD2 may exert its oncogenic roles by modulating the AKT signaling pathway. These findings may enrich the theoretical basis of MTHFD2-targeted therapy.

In recent years, MTHFD2 expression has been demonstrated to be increased in various types of cancer $(7,11)$, such as renal cell carcinoma, colorectal cancer, and its overexpression is considered as an independent prognostic signature in patients with breast cancer (14). In silico gene expression analysis carried out by Lehtinen et al (23) demonstrated that MTHFD2 overexpression is associated with metastasis of patients with breast cancer. Immunohistochemistry staining performed by Liu et al (14) also confirmed the patients with breast cancer patients and high expression of MTHFD2 experienced an unfavorable clinical outcome. Similarly to these two previous studies, the present study demonstrated that MTHFD2 expression was significantly increased in breast cancer tissues compared with adjacent normal tissues, according to the GEPIA database. In addition, OS analysis highlighted the potential of MTHFD2 as a prognostic biomarker for patients with breast cancer.

To further evaluate the oncogenic role of MTHFD2, functional assays were performed using MCF-7 cells. The results from the present study demonstrated that MTHFD2 overexpression increased MCF-7 cell proliferation and colony formation ability, whereas MTHFD2 knockout had an inhibitory effect on MCF-7 cell proliferation and colony formation ability. These results suggest that MTHFD2 may act as an oncogenic modulator in breast cancer cells, which is consistent with the results of a previous study on colorectal cancer (13). Lehtinen et al (23) also reported that RNAi-mediated silencing of MTHFD2 decreased migration and aggressiveness, resulting in decreased breast cancer stem cell properties in vivo. Koufaris et al (24) demonstrated that MTHFD2-knockdown MCF-7 cells presented with decreased proliferation and a slight decrease in their colony formation ability compared with parental cells after $72 \mathrm{~h}$. Similarly, the present study demonstrated that MTHFD2-deficient MCF-7 cells showed no significant colony formation after 3 days (data not shown). In the present study, there was a difference in colony formation ability between MTHFD2-deficient and normal MCF-7 cells after day 14. Off-target effects of the shRNA is also the possible reason to explain no impact on the colony formation shMTHFD2 caused. In addition, a significant difference in colon cancer xenograft tumor growth was observed between mice injected with MTHFD2-knockdown or control HCT-116 cells; however, no effects of MTHFD2-knockdown were detected in HCT-116 cell proliferation in vitro (24), which was not the case in the present study. This difference may be due to the choice of cell line.

It has been established that MTHFD2 functions as a critical enzyme in folate one-carbon metabolism and regulates numerous physiological processes in tumor cells, including nucleotide metabolism and amino acid interconversion (25). Its enzymatic role was also supported by a recent study demonstrating that MTHFD2 confers redox cofactor preference to mitochondrial NADPH, which is advantageous for defense against redox equilibrium failure and which therefore supports cell proliferation (6).

In addition to the enzymatic role of MTHFD2, increasing attention has been paid to the non-enzymatic functions of MTHFD2. The data from RNA profiling of NCI-H1299 cells following MTHFD2 silencing indicated that cycle-related genes, such as CCNA2, are overexpressed, implying that MTHFD2 might have no metabolic function (13). 


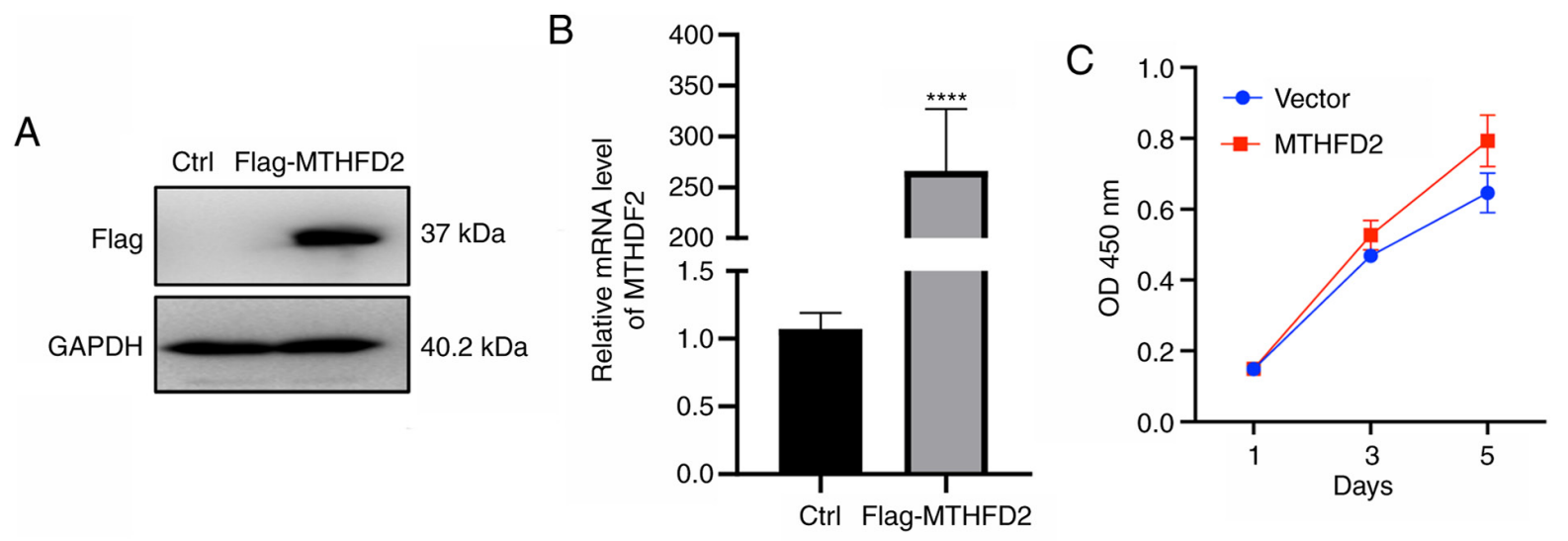

D
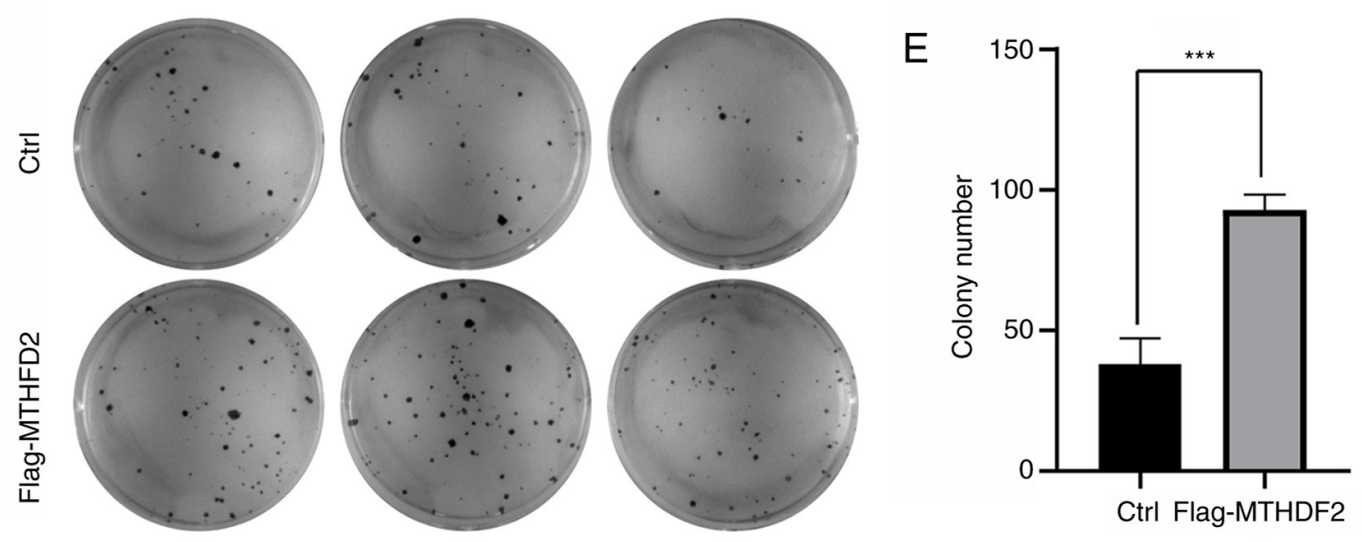

Figure 2. MTHFD2 overexpression increases the proliferation and clonogenicity of MCF-7 cells. (A) Western blotting and (B) reverse transcriptase quantitative PCR were performed to verify the efficacy of MTHFD2-Flag overexpression in MCF-7 cells. (C) Cell Counting Kit-8 assay was performed to examine MCF-7 cell proliferation following transfection with control vector and Flag-MTHFD2. (D and E) Colony formation assay was carried out to analyze the clonogenicity of cells transfected with control vector and Flag-MTHFD2. ${ }^{* * * *} \mathrm{P}<0.0001$. ${ }^{* * *} \mathrm{P}<0.001$. Ctrl, control; OD, optical density.

Furthermore, overexpression of MTHFD2 shows no reliance on its enzymatic activity to trigger tumor cell proliferative phenotypes in breast cancer cells (17). In addition, the nuclear localization of MTHFD2 has been demonstrated, suggesting that MTHFD2 may be a nuclear protein likely to be involved in the regulation of apoptosis and transcriptional events in order to facilitate tumor cell proliferation (17). These findings are supported by studies reporting that signal transduction pathways must participate in metabolic reprogramming to meet biosynthetic requirements and support tumor cell proliferation $(26,27)$. Furthermore, it has been reported that Pyrroline-5-carboxylate reductase 1, which is a mitochondrial metabolic enzyme, can regulate tumor cell phenotype by altering signaling pathways (28). The PI3K/AKT signaling pathway has a crucial role in cellular metabolism (25). In the present study, a luciferase reporter gene assay was therefore performed to examine the effect of MTHFD2 on the changes in transcriptional activity of AKT. As expected, the AKT signaling pathway was markedly activated upon MTHFD2 overexpression, indicating that MTHFD2 may regulate MCF-7 cell proliferation via the AKT signaling pathway.

AKT is an important molecule that senses numerous extracellular signals after being activated by various signal transduction cascades, including Bcr-Abl, Her2/neu and Ras (29). AKT activation has numerous effects to drive carcinogenesis of a number of types of cancers (29). Previous studies have reported that the AKT signaling pathway is a critical pathway by which tumor cell metabolism supports the metabolic autonomy of tumor cells $(30,31)$. It has been demonstrated that the AKT oncogene is responsible for the shift toward aerobic glycolysis, which is a distinct feature of metallic remodeling in tumor cells (29). The switch to aerobic glycolysis in tumor cells causes a high glycolytic rate and benefits cell survival by controlling mitochondrial homeostasis and preventing the activation of Bax (32). Increased AKT-induced metabolic alternations can also ensure a constant glycolysis and lactate production for both bioenergetics and biosynthesis to support tumor cell proliferation (33). In addition, AKT signaling can stimulate intracellular expression of nutrition transporter-associated proteins to maintain enough cell-autonomous nutrient uptake and capture (34). MTHFD2-mediated AKT signaling could therefore successfully regulate cell metabolism and signaling transduction, supporting therefore tumor cell self-renewal/proliferation (35). The findings from the present study strongly support the non-enzymatic role of nuclear MTHFD2 and explain why mutant MTHFD2, which lacks enzymatic activity, can still promote tumor cell proliferation. In the present study, activation of the AKT signaling pathway was decreased in MTHFD2 knockout MCF-7 cells. In addition, cell treatment with an AKT inhibitor inhibited the colonic formation ability caused by MTHFD2 overexpression. These results suggest that MTHFD2 overexpression activates the 
A

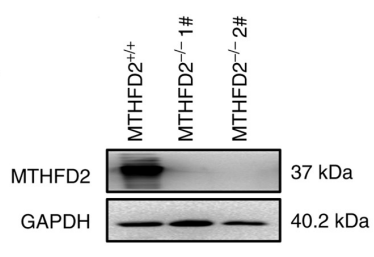

C

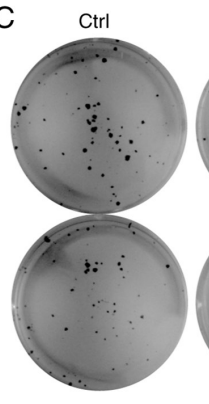

MTHFD2-1-1\#

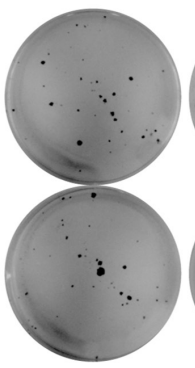

MTHFD2--2\#

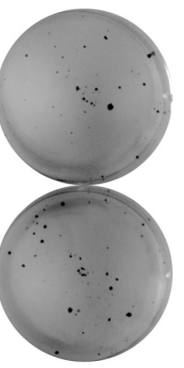

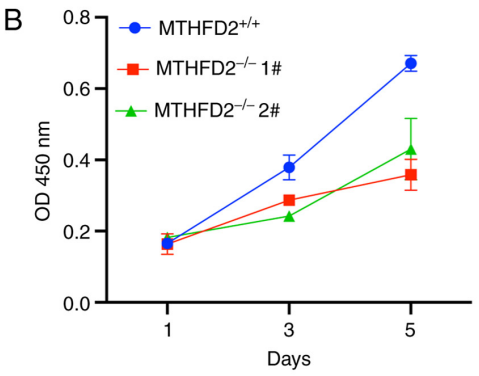

D

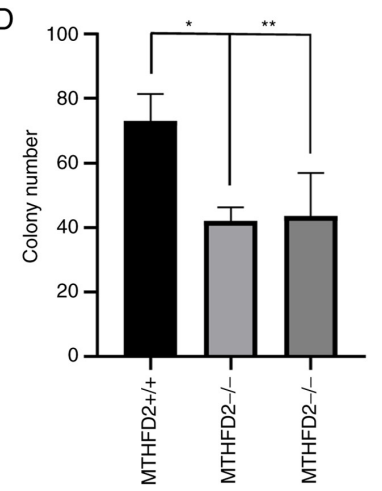

Figure 3. Genetic deletion of MTHFD2 impairs the proliferation and clonogenicity of MCF-7 cells. (A) MTHFD2 expression in MTHFD2-deficient and untransfected MCF-7 cells (Ctrl) was examined by western blotting. (B) Cell Counting Kit-8 assay was performed to examine the proliferation of MTHFD2-deficient MCF-7 cells. (C and D) Colony formation assay was carried out to analyze the clonogenicity of MTHFD2-deficient MCF-7 cells. "P<0.05 and $^{* *} \mathrm{P}<0.01$. OD, optical density.

A

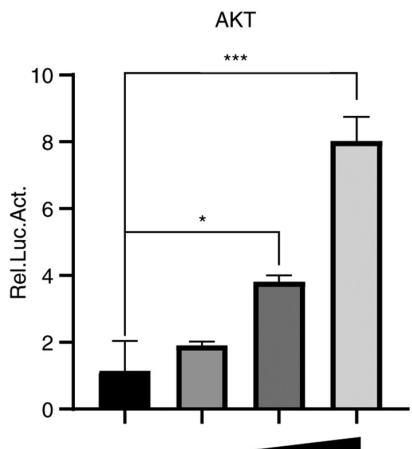

MTHFD2

C

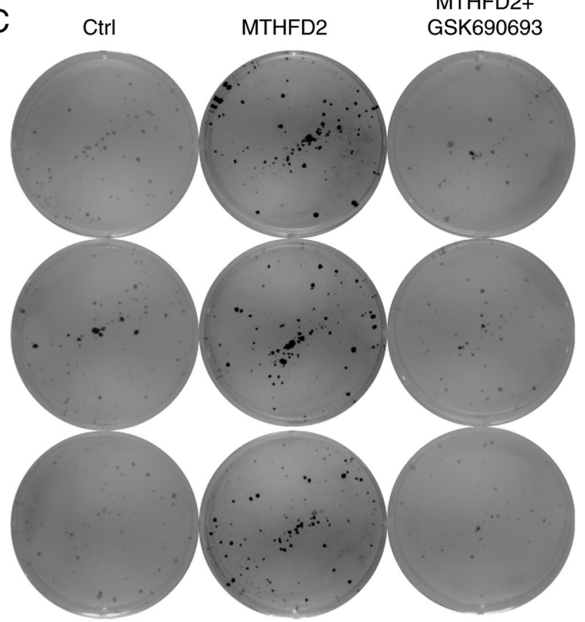

B

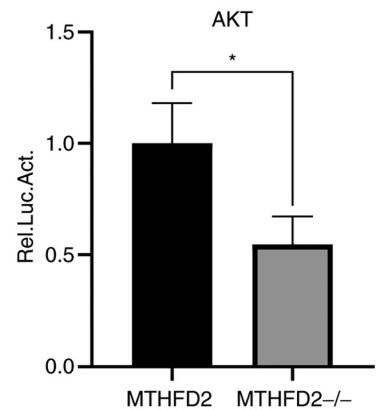

D

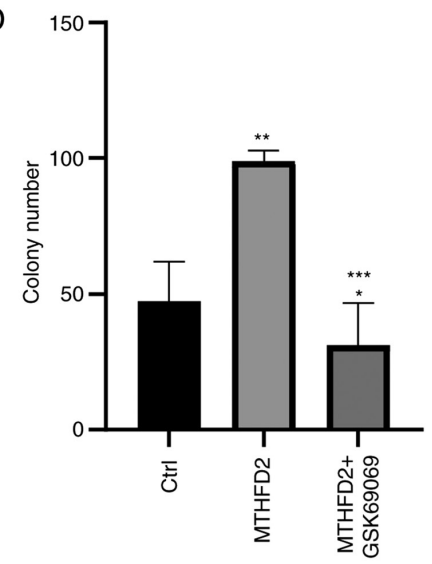

Figure 4. MTHFD2 stimulates AKT transcription and its MTHFD2 overexpression-induced colony formation of MCF-7 cells was impaired by AKT inhibitor (GSK6906). (A) Luciferase assays were performed to examine AKT transcriptional activity by co-transfecting 293T cells with an AKT luciferase reporter and different amounts of MTHFD2 plasmid. " $\mathrm{P}<0.05$ and ${ }^{* * *} \mathrm{P}<0.001$ vs. normal MCF-7 cells. (B) Luciferase assays showing AKT transcriptional activity in MTHFD2-knockdown MCF-7 cells. " $\mathrm{P}<0.05$ vs. MTHFD2-overexpressing cells. (C and D) Colony formation assay was carried out to analyze the clonogenicity of MTHFD2 overexpression on colony formation followed cell treatment with GSK69069. ${ }^{*} \mathrm{P}<0.05$ and ${ }^{* *} \mathrm{P}<0.01$ vs. Ctrl. ${ }^{* * *} \mathrm{P}<0.001$ vs. MTHFD2-overexpressing cells. Ctrl, control. 
AKT signaling pathway, enabling cancer cells to override the action of some limiting factors of cell proliferation in order to support biosynthesis metabolic phenotypes. However, AKT is not the only signaling pathway that is involved in the integrated metabolic reprogramming of tumor cells. For example, the Hippo pathway cascade has been demonstrated to be involved in cancer metabolic reprogramming (36). Future work will investigate the complex signaling networks mediated by MHTFD2 in breast cancer.

In summary, the present study demonstrated that the MTHFD2 expression level was increased in breast cancer tissues compared with adjacent normal tissues, and described its role on the stimulation of tumor cell proliferation via the AKT signaling pathway. This study demonstrated that MTHFD2 may increase proliferation of breast cancer cells via the AKT signaling pathway, complementing the suggestion of its enzymatic role in metabolic remodeling. These findings provide a theoretical basis for the use of MTHFD2 as a potential druggable target for breast cancer therapy. However, the lack of data from different cell lines is the main limitation of the present study. Future investigation is required to further determine the role of MTHFD2 in breast cancer.

\section{Acknowledgements}

Not applicable.

\section{Funding}

This work was supported by the Guangdong Medical Science and Technology Research Fund Project, China (grant no. C2019133).

\section{Availability of data and materials}

The datasets used and/or analyzed during the current study are available from the corresponding author on reasonable request.

\section{Authors' contributions}

JH, YQ and CL drafted the manuscript and revised it critically for important intellectual content, acquired, analyzed and interpreted the data. XH has been involved in drafting the manuscript and interpreting the data. FZ made substantial contributions to conception and design of the present study. All authors read and approved the final version of the manuscript.

\section{Ethics approval and consent to participate}

Not applicable.

\section{Patient consent for publication}

Not applicable.

\section{Competing interests}

The authors declare that they have no competing interests.

\section{References}

1. Mattiuzzi C and Lippi G: Current cancer epidemiology. J Epidemiol Glob Health 9: 217-222, 2019.

2. Siegel RL, Miller KD and Jemal A: Cancer statistics, 2018. CA Cancer J Clin 68: 7-30, 2018.

3. DeSantis CE, Ma J, Gaudet MM, Newman LA, Miller KD, Goding Sauer A, Jemal A and Siegel RL: Breast cancer statistics, 2019. CA Cancer J Clin 69: 438-451, 2019.

4. Ito $\mathrm{H}$ and Matsuo $\mathrm{K}$ : Molecular epidemiology, and possible real-world applications in breast cancer. Breast Cancer 23: 33-38, 2016.

5. Ghoncheh M, Pournamdar Z and Salehiniya H: Incidence and mortality and epidemiology of breast cancer in the world. Asian Pac J Cancer Prev 17: 43-46, 2016.

6. Shin M, Momb J and Appling DR: Human mitochondrial MTHFD2 is a dual redox cofactor-specific methylenetetrahydrofolate dehydrogenase/methenyltetrahydrofolate cyclohydrolase. Cancer Metab 5: 11, 2017.

7. Green NH, Galvan DL, Badal SS, Chang BH, LeBleu VS, Long J, Jonasch E and Danesh FR: MTHFD2 links RNA methylation to metabolic reprogramming in renal cell carcinoma. Oncogene 38: 6211-6225, 2019.

8. Nilsson R, Jain M, Madhusudhan N, Sheppard NG, Strittmatter L, Kampf C, Huang J, Asplund A and Mootha VK: Metabolic enzyme expression highlights a key role for MTHFD2 and the mitochondrial folate pathway in cancer. Nat Commun 5: 3128 , 2014.

9. Mattaini KR, Sullivan MR and Vander Heiden MG: The importance of serine metabolism in cancer. J Cell Biol 214: 249-257, 2016.

10. Ju HQ, Lu YX, Chen DL, Zuo ZX, Liu ZX, Wu QN, Mo HY, Wang ZX, Wang DS, Pu HY, et al: Modulation of redox homeostasis by inhibition of MTHFD2 in colorectal cancer: Mechanisms and therapeutic implications. J Natl Cancer Inst 111: 584-596, 2019.

11. Lin H, Huang B, Wang H, Liu X, Hong Y, Qiu S and Zheng J: MTHFD2 overexpression predicts poor prognosis in renal cell carcinoma and is associated with cell proliferation and vimentin-modulated migration and invasion. Cell Physiol Biochem 51: 991-1000, 2018.

12. Pikman Y, Puissant A, Alexe G, Furman A, Chen LM, Frumm SM, Ross L, Fenouille N, Bassil CF, Lewis CA, et al: Targeting MTHFD2 in acute myeloid leukemia. J Exp Med 213: 1285-1306, 2016.

13. Yu C, Yang L, Cai M, Zhou F, Xiao S, Li Y, Wan T, Cheng D, Wang L, Zhao C and Huang X: Down-regulation of MTHFD2 inhibits NSCLC progression by suppressing cycle-related genes. J Cell Mol Med 24: 1568-1577, 2020.

14. Liu F, Liu Y, He C, Tao L, He X, Song H and Zhang G: Increased MTHFD2 expression is associated with poor prognosis in breast cancer. Tumour Biol 35: 8685-8690, 2014.

15. Tedeschi PM, Vazquez A, Kerrigan JE and Bertino JR: Mitochondrial methylenetetrahydrofolate dehydrogenase (MTHFD2) overexpression is associated with tumor cell proliferation and is a novel target for drug development. Mol Cancer Res 13: 1361-1366, 2015.

16. Wei Y, Liu P, Li Q, Du J, Chen Y, Wang Y, Shi H, Wang Y, Zhang H, Xue W, et al: The effect of MTHFD2 on the proliferation and migration of colorectal cancer cell lines. Onco Targets Ther 12: 6361-6370, 2019.

17. Sheppard NG, Jarl L, Mahadessian D, Strittmatter L, Schmidt A, Madhusudan N, Tegnér J, Lundberg EK, Asplund A, Jain M and Nilsson R: The folate-coupled enzyme MTHFD2 is a nuclear protein and promotes cell proliferation. Sci Rep 5: 15029, 2015.

18. Derunes C, Burgess R, Iraheta E, Kellerer R, Becherer K, Gessner CR, Li S, Hewitt K, Vuori K, Pasquale EB, et al: Molecular determinants for interaction of SHEP1 with Cas localize to a highly solvent-protected region in the complex. FEBS Lett 580: 175-178, 2006.

19. Wigler M, Pellicer A, Silverstein S and Axel R: Biochemical transfer of single-copy eucaryotic genes using total cellular DNA as donor. Cell 14: 725-731, 1978.

20. Livak KJ and Schmittgen TD: Analysis of relative gene expression data using real-time quantitative PCR and the 2(-Delta Delta C(T)) method. Methods 25: 402-408, 2001.

21. Liang T, Zhang Y, Yin S, Gan T, An T, Zhang R, Wang Y, Huang Y, Zhou Q and Zhang J: Cardio-protecteffect of qiliqiangxin capsule on left ventricular remodeling, dysfunction and apoptosis in heart failure rats after chronic myocardial infarction. Am J Transl Res 8: 2047-2058, 2016. 
22. Tang Z, Li C, Kang B, Gao G, Li C and Zhang Z: GEPIA: A web server for cancer and normal gene expression profiling and interactive analyses. Nucleic Acids Res 45: W98-W102, 2017.

23. Lehtinen L, Ketola K, Mäkelä R, Mpindi JP, Viitala M, Kallioniemi $\mathrm{O}$ and Iljin K: High-throughput RNAi screening for novel modulators of vimentin expression identifies MTHFD2 as a regulator of breast cancer cell migration and invasion. Oncotarget 4 : 48-63, 2013.

24. Koufaris C, Gallage S, Yang T, Lau CH, Valbuena GN and Keun HC: Suppression of MTHFD2 in MCF-7 breast cancer cells increases glycolysis, dependency on exogenous glycine, and sensitivity to folate depletion. J Proteome Res 15: 2618-2625, 2016.

25. DeBerardinis RJ, Lum JJ, Hatzivassiliou G and Thompson CB: The biology of cancer: Metabolic reprogramming fuels cell growth and proliferation. Cell Metab 7: 11-20, 2008.

26. Campbell SL and Wellen KE: Metabolic signaling to the nucleus in cancer. Mol Cell 71: 398-408, 2018.

27. Torti SV and Torti FM: Iron and cancer: More ore to be mined. Nat Rev Cancer 13: 342-355, 2013.

28. Zhuang J, Song Y, Ye Y, He S, Ma X, Zhang M, Ni J, Wang J and Xia W: PYCR 1 interference inhibits cell growth and survival via c-Jun N-terminal kinase/insulin receptor substrate 1 (JNK/IRS1) pathway in hepatocellular cancer. J Transl Med 17: 343, 2019.

29. Elstrom RL, Bauer DE, Buzzai M, Karnauskas R, Harris MH, Plas DR, Zhuang H, Cinalli RM, Alavi A, Rudin CM and Thompson CB: Akt stimulates aerobic glycolysis in cancer cells. Cancer Res 64: 3892-3899, 2004.

30. Chu N, Salguero AL, Liu AZ, Chen Z, Dempsey DR, Ficarro SB, Alexander WM, Marto JA, Li Y, Amzel LM, et al: Akt kinase activation mechanisms revealed using protein semisynthesis Cell 174: 897-907.e14, 2018.
31. Schultze SM, Hemmings BA, Niessen $M$ and Tschopp O: PI3K/AKT, MAPK and AMPK signalling: Protein kinases in glucose homeostasis. Expert Rev Mol Med 14: e1, 2012.

32. Rathmell JC, Fox CJ, Plas DR, Hammerman PS, Cinalli RM and Thompson CB: Akt-directed glucose metabolism can prevent Bax conformation change and promote growth factorindependent survival. Mol Cell Biol 23: 7315-7328, 2003.

33. Tong $\mathrm{X}$, Zhao $\mathrm{F}$ and Thompson CB: The molecular determinants of de novo nucleotide biosynthesis in cancer cells. Curr Opin Genet Dev 19: 32-37, 2009.

34. Wieman HL, Wofford JA and Rathmell JC: Cytokine stimulation promotes glucose uptake via phosphatidylinositol-3 kinase/Akt regulation of Glut1 activity and trafficking. Mol Biol Cell 18: 1437-1446, 2007.

35. Vivanco I and Sawyers CL: The phosphatidylinositol 3-Kinase AKT pathway in human cancer. Nat Rev Cancer 2: 489-501, 2002.

36. Zhang X, Zhao H, Li Y, Xia D, Yang L, Ma Y and Li H: The role of YAP/TAZ activity in cancer metabolic reprogramming. Mol Cancer 17: 134, 2018.

This work is licensed under a Creative Commons Attribution-NonCommercial-NoDerivatives 4.0 International (CC BY-NC-ND 4.0) License. 